\title{
Coexistence Problem in IEEE 802.22 Wireless Regional Area Networks
}

\author{
Raed Al-Zubi, Mohammad Z. Siam, and Marwan Krunz \\ Department of Electrical and Computer Engineering \\ University of Arizona, Tucson, AZ 85721 \\ E-mail: \{alzubi, siam, krunz\}@ece.arizona.edu
}

\begin{abstract}
IEEE 802.22 wireless regional area network (WRAN) is an emerging cognitive radio-based system. One of the major challenges for WRANs is how to efficiently schedule both channel sensing and data transmission for multiple adjacent WRAN cells. This challenge is known as coexistence problem. In this paper, we propose four schemes that aim at reducing the coexistence-problem effect. These schemes are based on a well-known operation mode of IEEE 802.22, namely dynamic frequency hopping (DFH). The first and second schemes are based on using omni-directional antennas at the base stations (BSs), whereas the BSs in the other two schemes use directional antennas. The first scheme, coined fixed-scheduling DFH (FDFH), bases upon a fixed scheduling of working channels for adjacent WRAN cells. The second scheme, called cooperative DFH (CDFH), cooperatively selects working channels. The third scheme, namely sectoral DFH (SDFH), is proposed to reduce the coordination overhead of CDFH via dividing a WRAN cell into sectors to decrease the chances of collisions between adjacent cells. Finally, we integrate FDFH and SDFH into a new scheme, called fixed-scheduling sectoral DFH (FSDFH), which exploits the advantages of both schemes with no additional overhead. Computer simulations are used to demonstrate the performance gain of the proposed schemes.
\end{abstract}

\section{INTRODUCTION}

IEEE 802.22 wireless regional area network (WRAN) is a new type of wireless networks that is expected to be widely used in the future. Being a new system that has not been officially standardized yet, several open issues regarding WRAN's operations still exist. The IEEE 802.22 working group (WG) [1] has been formed in November 2004 to develop a standard for WRANs, based on cognitive radio technology. This WG includes several known corporations (e.g., Philips, Intel, Motorola, ST Micro, CRC, Samsung, and Nokia) as well as delegates from the incumbent world (e.g., Fox, CBS, NAB, MSN, and Shure Inc.). IEEE 802.22 WRAN systems operate on the VHF/UHF TV bands ranging from $54 \mathrm{MHz}$ to 862 MHz. The main target of WRANs is to provide wireless broadband access (e.g., DSL service in rural areas). The average coverage radius of a WRAN cell is $33 \mathrm{Km}$, and can go up to $100 \mathrm{Km}$.

One of the major challenges for WRAN systems is how to efficiently schedule both channel sensing and data transmission, as a channel cannot be simultaneously used for sensing and data transmission [2]. The nonhopping mode, which is the basic mode of IEEE 802.22 systems [3], requires that a WRAN cell operating on a single channel should interrupt data transmissions every 2 seconds for sensing [4]. This periodic interruption decreases the system throughput and can significantly affect the quality of service (QoS) for IEEE 802.22 systems (e.g., voice transmissions can tolerate up to 20 ms interruption).

One of the recently proposed schemes that address this problem is the dynamic-frequency-hopping (DFH) scheme [3]. In this scheme, data transmission is performed using one of the available channels, while the other channels are simultaneously sensed. After 2 seconds, a WRAN cell hops to a new working channel and vacates the previously used one. To achieve efficient performance of DFH, multiple adjacent WRAN cells have to coordinate their hopping behavior. This coordination reduces the effect of a well-known problem in WRAN systems, namely coexistence problem. A contention-based scheme [5] was proposed to solve this problem. This scheme jointly handles channel on demand between BSs belonging to a same operator (intra-operator situation) and channel on demand between BSs belonging to different operators (inter-operators situation). However, this scheme results in significant overhead and delay (100 Km range imposes a round-trip propagation delay of about $300 \mu \mathrm{sec})$. This is due to the fact that three messages (channel contention request, channel contention reply, and channel contention acknowledgement) have to be exchanged at each time that a channel needs to be reserved.

In this paper, we propose several variants of the DFH scheme that aim at reducing the coexistence-problem effect. The first scheme is called fixed-scheduling $D F H$ $(F D F H)$. The key idea behind this scheme is that neighboring WRAN cells determine a fixed schedule for selecting the next working channel. To overcome the static nature of this scheme, we propose another scheme, namely cooperative $D F H(C D F H)$, that cooperatively selects working channels. As the latter scheme requires coordination overhead between BSs, we are motivated to propose an overhead-free scheme, called sectoral DFH $(S D F H)$. SDFH divides a WRAN cell into sectors to decrease the chances of having several WRAN users in overlapped sectors between neighboring BSs that are served by same operating channels. Finally, we integrate FDFH and SDFH into a new scheme, called fixedscheduling sectoral DFH (FSDFH), which exploits the advantages of both schemes with no additional overhead.

The rest of this paper is organized as follows. We overview the basic preliminaries of IEEE 802.22 in Section II. We discuss the operational details of DFH, FDFH, CDFH, SDFH, and FSDFH in Sections III, IV, and V, VI, VII, respectively. We compare the performance of the proposed schemes in Section VIII. Finally, concluding remarks and suggested future work are provided in Section IX.

\section{OVERVIEW OF IEEE 802.22}

In this section, we overview the basic preliminaries of IEEE 802.22. We start with highlighting the specifications of a WRAN system. We then briefly present its main applications. Finally, we explain the coexistence problem.

\section{A. Specifications}

According to the IEEE 802.22 WG [1], the operation mode of WRAN systems should be point to multi-point. 
In each WRAN cell, the customer-premises equipments (CPEs) are attached to a BS via a wireless link using the available (not used by a primary user, i.e., TV station or wireless microphone) $\mathrm{VHF} / \mathrm{UHF} \mathrm{TV}$ bands ranging from $54 \mathrm{MHz}$ to $862 \mathrm{MHz}^{1}$. The sensing process that is used to discover the available spectrum could be done in a centralized or a distributed manner. For the centralized approach, centralized servers inform CPEs about the available TV channels. The distributed approach allows local spectrum sensing only, where each CPE decides by itself which channels are available for a communication. A combination of these two approaches is also envisioned.

The physical layer in IEEE 802.22 should have the ability to adapt itself according to the surrounding environment. The BSs and CPEs should be flexible to jump between channels without causing errors in transmissions, and adjust their bandwidth, modulation, and coding schemes. All these adaptive processes are controlled by the BS of a cell. Orthogonal frequency division multiple access (OFDMA) is the modulation scheme to be used for transmission in up/down links.

Various specifications of the IEEE 802.22 WRAN system are given in Table I [7].

\begin{tabular}{|c|c|}
\hline RF channel bandwidth & $6 \mathrm{MHz}$ \\
\hline Average spectrum efficiency & $3 \mathrm{bits} / \mathrm{s} / \mathrm{Hz}$ \\
\hline Channel capacity & $18 \mathrm{Mbps}$ \\
\hline System capacity per subscriber (forward) & $1.5 \mathrm{Mbps}$ \\
\hline System capacity per subscriber (backward) & $384 \mathrm{kbps}$ \\
\hline Forward/backward ratio & 3.9 \\
\hline Over-subscription ratio & 50 \\
\hline Number of subscribers per forward channel & 600 \\
\hline Minimum number of subscribers & 90 \\
\hline Early take-up rate & $3 \mathrm{bits} / \mathrm{s} / \mathrm{Hz}$ \\
\hline Potential number of subscribers & 1800 \\
\hline Number of persons per household & 2.5 \\
\hline Total number of persons per coverage area & 4500 \\
\hline BS effective-isotropic-radiated power & $98.3 \mathrm{~W}$ \\
\hline Minimum population density covered & $1.5 \mathrm{persons} / \mathrm{km}^{2}$ \\
\hline
\end{tabular}

TABLE I

\section{B. Applications}

IEEE 802.22 SPECIFICATIONS.

The most significant application of IEEE 802.22 is to provide rural and remote regions with wireless broadband access. The significance of such an application comes from the fact that about half of the population in USA exists in rural and remote areas [8]. The same situation applies to other regions, e.g., Africa, Asia, etc. It should be noted that the applicability of IEEE 802.22 is not restricted to such rural regions. As a matter of fact, other targets of IEEE 802.22 applications include single-family residential, multi-dwelling units, small businesses, multitenant buildings, public and private campuses, etc. Data, voice, and video traffic with appropriate QoS are also examples of services that IEEE 802.22 supports.

\section{Coexistence Problem}

As shown in Figure 1, several WRAN cells may overlap in their working vicinities. The resultant interference between these overlapped cells leads to one of the

\footnotetext{
${ }^{1}$ In the USA, TV stations use channels 2 to 69 in the VHF and UHF portions of the radio spectrum. All these channels are $6 \mathrm{MHz}$ wide, and span from $54-72 \mathrm{MHz}, 76-88 \mathrm{MHz}, 174-216 \mathrm{MHz}$, and $470-806$ $\mathrm{MHz}[6]$.
}

major challenges in WRAN systems, namely coexistence problem. This problem may degrade the performance of the system due to the fact that the WRAN coverage range can go up to $100 \mathrm{Km}$. As a result, the interference range of this WRAN cell is larger than that in any existing unlicensed technology. Furthermore, a WRAN system opportunistically operates in an unlicensed spectrum, unlike other systems such as cellular systems in which operators use a certain portion of their licensed spectrum. Therefore, coordination between different BSs is needed.

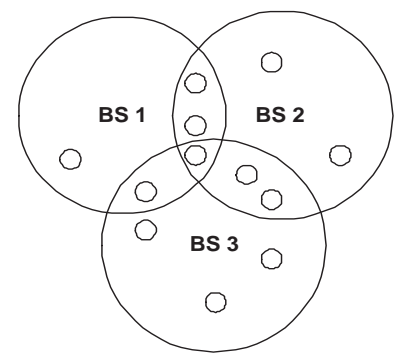

Fig. 1. Coexistence problem in IEEE 802.22.

Unlike other IEEE 802 standards where coexistence issues are often considered only after the standard is issued, the IEEE 802.22 WG requires that the air interface includes coexistence protocols and algorithms as part of the standard.

\section{THE DFH SCHEME}

In this section, we overview the DFH scheme, which represents the base for our proposed schemes.

The rationale behind proposing the DFH scheme is to overcome the main problem of the first proposed scheme for IEEE 802.22, namely the non-hopping scheme. This problem is that the non-hopping scheme limits the simultaneous operation of both sensing and data transmission. What makes this problem worse is that any working channel has to be periodically (each 2 seconds) vacated for sensing. This periodic vacation degrades the system performance, as several delay-sensitive applications cannot tolerate this interruption.

The operation of the DFH scheme can be summarized as follows. A WRAN cell in the DFH scheme uses the available channels for data transmissions. It simultaneously performs spectrum sensing on all other channels. This operation is known as simultaneous sensing and data transmissions (SSDT). To allow for such operation, the time axis is divided into consecutive working periods, in each of which a WRAN cell is operating on the available channels, while simultaneously sensing all other channels, as shown in Figure 2. As a result, a WRAN system exploiting DFH dynamically selects one of the available channels in the current operation period as a next working channel for data transmission. It should be noted that this channel can only be used for data transmission for at most 2 seconds [4] after the time it was validated.

One requirement for efficient operation of $\mathrm{DFH}$ is to execute the channel switching operation fast enough. Given that in today's technologies the delay of such a switching is small enough, this requirement can be fulfilled. For example, this delay is in the range of tens of microseconds in the current IEEE 802.11 wireless cards [9].

The main concern about the DFH scheme is that it does not consider the hopping behavior of adjacent WRAN cells, which yields the previously discussed problem (coexistence problem). 


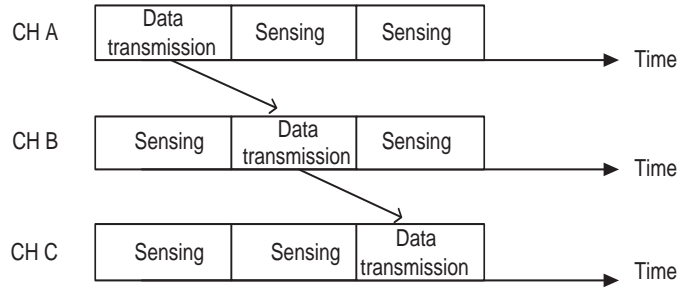

Fig. 2. Basic operation of the DFH scheme.

\section{THE FDFH SCHEME}

In this section, we discuss the operational details of our proposed FDFH scheme, which aims at mitigating the effect of the coexistence problem. Hence, improving the overall system performance. This improvement is seen in terms of increasing the number of served users in the overlapped areas between adjacent cells via reducing the chances of collisions in such areas.

The FDFH scheme can also serve as a preliminary stage in other protocols that try to solve the coexistence problem. For instance, this scheme can be used as a primary stage in the contention-based scheme discussed in section I. This integration reduces the significant delay and overhead that the contention-based scheme results in. Another example is the integration of FDFH into our proposed FSDFH scheme (we will explain the details of FSDFH in Section VII).

The key idea behind the FDFH scheme is that each WRAN cell gets a fixed schedule for its working channels. The operation of the proposed FDFH scheme is described via the following example. Consider a WRAN system with three BSs, as shown in Figure 3.

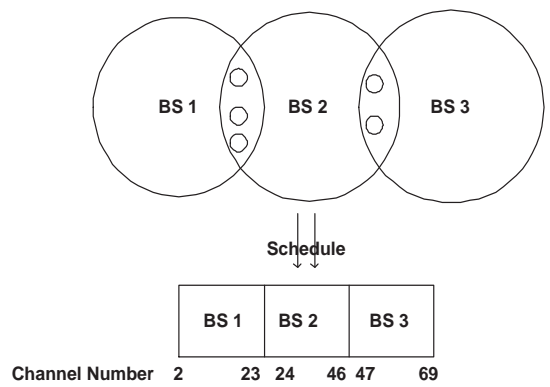

Fig. 3. Example illustrating the FDFH scheme (67 TV channels in USA).

First of all, the schedule of working channels for each WRAN cell in the system is determined. Since this schedule is fixed for each cell, it is determined and provided to the cells during the design (setup) phase. This schedule is updated due to any future change (e.g., a BS is added) in the system.

In our example, BS1 selects its next working channel among channels 2 to 23 , starting from channel 2 . If BS1 finds an available channel in this range, it uses this channel as its next working channel. Otherwise, it searches for an available channel in the range of channels that are given to BS3, i.e., channels 47 to 69 . If BS1 still does not find an available channel, it searches in the range of channels that are given to BS2, i.e., channels 24 to 46 (starting from channel 46 to reduce the chances of selecting the same channel that BS2 may choose). BS2 and BS3 apply the same procedure.

It is clear from this example that the FDFH scheme decreases the chances of collisions that may occur in the overlapped areas between adjacent BSs. As a result, this increases the number of served users in the overlapped areas, which eventually decreases the effect of the coexistence problem. It should be noted that the proposed FDFH scheme has the following features: Simplicity, no additional hardware is required, low overhead, and no synchronization technique is needed between adjacent BSs.

\section{THE CDFH SCHEME}

In this section, we present our proposed $\mathrm{CDFH}$ scheme. The motivation behind proposing such a scheme is to overcome the static nature of FDFH. The key idea of CDFH is that each WRAN cell cooperatively selects its working channels, taking into account the working channels of its neighboring cells.

According to this scheme, time is divided into 2second intervals (including a beacon interval (BI)), as shown in Figure 4. During the BI, a BS, say $X$, sends a beacon at a high-power value over all available channels that are not occupied by primary users. Each BS is equipped with multiple transceivers to allow for simultaneously sending/receiving beacons over all available channels. Although it may seem that this feature requires additional hardware, it is only required at BSs, whose number is limited and lasts for long time. Based on using the high-power value for sending beacons, these beacons can be received by other BSs whose transmission ranges overlap with that of $X$. Accordingly, the transmission range of the beacon is selected as twice that of data (which is $33 \mathrm{Km}$ ), i.e., a beacon's transmission range $=66 \mathrm{Km}$. Each beacon includes the following fields: (1) The available channels that will be used in the rest of the 2-second interval, (2) a list of users that lie in the BS's data-transmission range, (3) the BS's location (assuming that each BS is equipped with a GPS), and (4) the time schedule of sending beacons by adjacent BSs (i.e., the sequence of beacon transmissions). Note that once a BS hears a beacon sent by other BSs, it decides if it is supposed to transmit a beacon in the next slot (based on the time schedule of beacon transmissions and the receipt time of the received beacon).

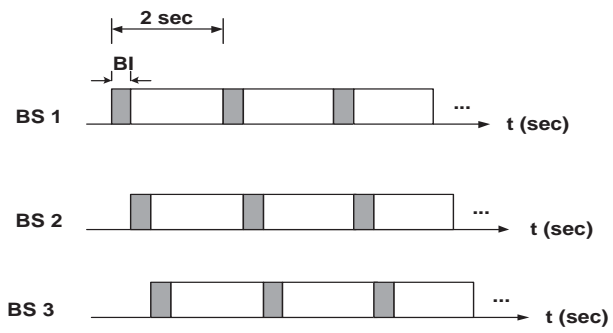

Fig. 4. Time structure of the CDFH scheme.

We now explain the main components of the CDFH scheme, which are: Synchronization, listening and sensing, and channels selection.

\section{A. Synchronization}

The CDFH scheme requires synchronization between adjacent BSs. This requirement is fulfilled via transmitting beacons, where each newly established BS has to scan all available channels (before starting its operation) to detect all beacons that have been transmitted by other adjacent BSs. Accordingly, each newly established BS adds itself to the current schedule of beacons' transmissions, and announces the updated schedule via an upcoming beacon. 
The above operation is exemplified in Figure 5. Consider three $\operatorname{BSs}(A, B$, and $C)$ that are already in operation. The time schedule of sending beacons for $A$ and $B$ is as follows: $A$ sends its beacon first, then $B$ sends its beacon after it hears $A$ 's beacon. The time schedule of $C$ only contains itself, as $C$ cannot hear any of $A$ 's or $B$ 's beacons. Assume that a new BS, say $D$, has been established between $B$ and $C$. According to the time schedule for beacons' transmissions that is announced by $B$ and $C, D$ announces an updated schedule (e.g., $D$ may announce the following time sequence for sending beacons: $A$ followed by $B$, then $C$, and finally $D$ ). As a result, $C$ synchronizes its beacon transmission with this new schedule.

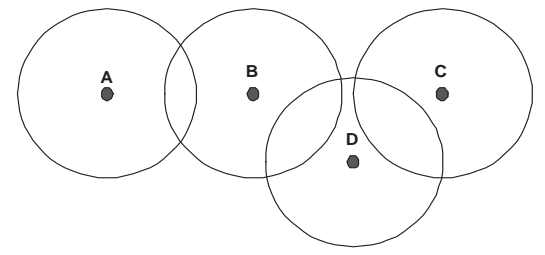

Fig. 5. Example illustrating synchronization between BSs in CDFH.

\section{B. Listening and Sensing}

The second component of $\mathrm{CDFH}$ is the listening process. The purpose of this process is to detect all the beacons that have been sent by adjacent BSs. Once these beacons are detected, the other processes of CDFH (synchronization and channels selection) can be performed.

It should be noted that this listening process differs from the sensing process. The purpose of the sensing process is to determine the available channels that are not occupied by primary users. In our work, we assume that the sensing process is done in a way similar to that in $\mathrm{DFH}$, where two ways (centralized and distributed) can be used. In the former one, centralized servers inform the CPEs about the available channels. In the distributed way, each CPE performs local spectrum sensing to decide (by itself) which channels are available for communication. Note that a combination of these two ways is also possible.

\section{Channels Selection and Reuse}

In order for a BS to announce the selected working channels via its beacon at the beginning of each 2 -second interval, it has to consider the results of the listening and sensing processes. According to the results of the sensing process, a BS should avoid using the channels that are occupied by primary users. Based on the results of the listening process, the BS can use the channels that are not occupied by its adjacent BSs that have common users (in the overlapped area) with this BS.

According to the proposed CDFH scheme, a BS has the ability to reuse the channels that are occupied by its adjacent BSs, as illustrated in the following example. Consider Figure 6 , where BS $A$ can reuse the channels that are occupied by the other BS $(B)$ to serve the users that lie in the transmission range $R_{2}$. Note that this range can be easily calculated by $A$, as it knows the location and transmission range of $B$ (which are announced by $B$ in its beacon).

\section{THE SDFH SCHEME}

In this section, we present the third proposed scheme, namely sectoral dynamic frequency hopping (SDFH). The key feature of SDFH is that it divides a WRAN cell

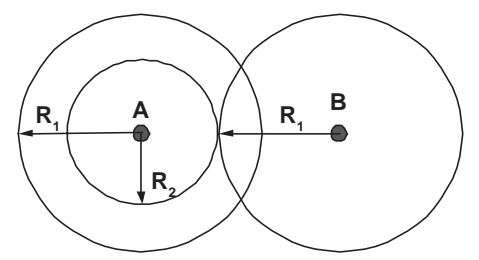

Fig. 6. Example of channel reuse in CDFH.

into sectors. Using such an idea decreases the chances of collisions in the overlapped areas between adjacent WRAN cells, which increases the number of served users in the overlapped areas. This is attributed to the fact that each BS uses directional antennas to serve users in each sector (instead of using omni-directional antennas, as in $\mathrm{DFH}$ ). This results in having smaller overlapping regions, which leads to low number of users that are affected by the coexistence problem.

The key idea behind the SDFH scheme is explained via the following example. Consider Figure 7 that consists of two neighboring BSs ( $X$ and $Y$ ), which overlap in five nodes $(A, B, C, D$, and $E)$. BS $X$ is responsible for serving nodes $B$ and $E$, while $\mathrm{BS} Y$ is responsible for serving nodes $A, C$, and $D$. Assume that channels 1 and 2 are available for both $X$ and $Y$. BS $X$ serves node $B$ with channel 2 and node $E$ with channel 1 . For BS $Y$, node $A$ is served by channel 1 , whereas nodes $C$ and $D$ are served by channel 2 . According to the DFH scheme, none of the five nodes is served, as both BSs use the same operating channels in the overlapped area (channel 1 is used to serve nodes $A$ and $E$, and channel 2 is used to serve nodes $B, C$, and $D$ ).

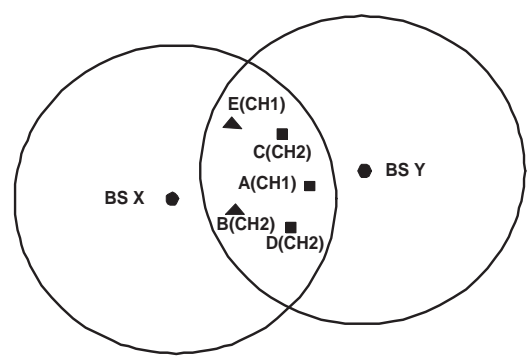

Fig. 7. Example illustrating the superiority of SDFH over DFH.

Figure 8 explains the operation of SDFH. The same setup (node locations, available channels, etc.) of Figure 7 applies here. According to the sectoring feature of SDFH, nodes $B$ and $D$ lie in an overlapped sector between $X$ and $Y$, and they are served by the same channel (channel 2). Thus, none of them is served. However, nodes $A, C$, and $E$ are served, as they lie in sectors that do not use the same channels for both BSs. By comparing the achieved service in Figure 8 with that in Figure 7, we see that SDFH results in more served users (three out of five nodes are successfully served) in the overlapped areas between the neighboring BSs compared with DFH (none of the five nodes is successfully served).

\section{THE FSDFH SCHEME}

In this section, we integrate the features of FDFH and SDFH into a new proposed scheme, called fixedscheduling sectoral dynamic frequency hopping (FSDFH). This integration exploits the attractive features of both FDFH and SDFH. In particular, both of them do not require coordination overhead between BSs compared 


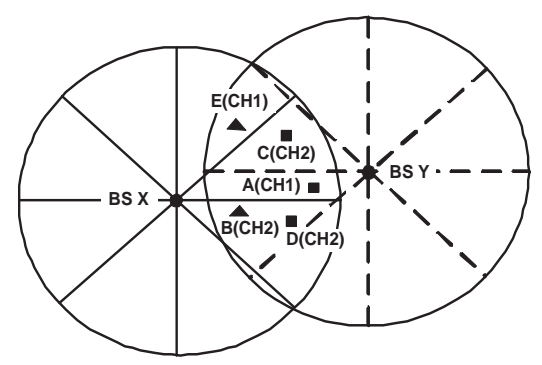

Fig. 8. Example illustrating the SDFH scheme.

with CDFH. An interesting feature of this integration is that it does not result in additional complexity overhead compared with any of the SDFH and SDFH schemes.

It is worth mentioning that integrating FDFH into this new scheme (FSDFH) lends credence to our claim that FDFH can be used (with no additional overhead) as a preliminary stage for other proposed schemes that try to solve the coexistence problem.

Both the process of looking for available channels in FDFH and the sectoring feature of SDFH result in decreasing the probability of having several WRAN users in overlapped sectors between neighboring BSs that are served by same operating channels. As a result, FSDFH is expected to decrease the chances of collisions in the overlapped areas. Hence, FSDFH mitigates the impact of the coexistence problem on the performance of WRAN systems.

\section{PERformance EVAluation}

\section{A. Simulation Setup}

In this section, we evaluate the performance of our proposed schemes (FDFH, CDFH, SDFH, and FSDFH), and compare it with that of the fundamental operation mode of IEEE 802.22 (DFH). Our results are based on simulation experiments conducted using CSIM programs (CSIM is a C-based process-oriented discrete-event simulation package [10]). We consider a WRAN system that consists of $4 \mathrm{BSs}$ that are randomly located in a square area of $150 \mathrm{Km} \mathrm{x} 150 \mathrm{Km}$. The users are uniformly distributed over the four WRAN cells. A summary of the simulation parameters is given in Table II. These parameters correspond to realistic hardware settings and follow the draft standard of the IEEE 802.22 [3].

\begin{tabular}{|c|c|}
\hline Number of BSs & 4 \\
\hline BS range & $33 \mathrm{Km}$ \\
\hline Number of served users per channel & 12 \\
\hline Operation slot time for a WRAN cell & $2 \mathrm{sec}$ \\
\hline Network area & $150 \mathrm{Km} \mathrm{x} \mathrm{150} \mathrm{Km}$ \\
\hline Total channel capacity & $18 \mathrm{Mbps}$ \\
\hline Channel capacity per user & $1.5 \mathrm{Mbps}$ \\
\hline Number of primary channels & 67 \\
\hline
\end{tabular}

TABLE II

SiMULATION PARAMETERS.

\section{B. Simulation Results}

Our main goal in this paper is to mitigate the coexistence-problem impact on the performance of the overlapped areas between adjacent BSs. Therefore, we focus in this section on the performance in the overlapped areas between adjacent BSs in terms of throughput,

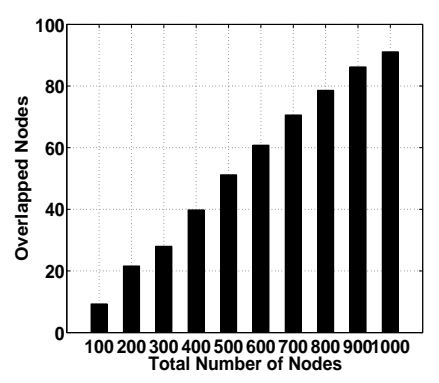

Fig. 9. Number of overlapped users versus total number of users.

number of served users, and bit-error rate (BER). We evaluate the performance of our proposed schemes under sparse and dense topologies.

1) Performance under a Sparse Network: In this case, we consider a sparse topology, where the total number of users ranges from 100 to 1000 users.

In Figure 9, we study the impact of the network density (number of users per square meter) on the coexistence problem. This figure reveals that the number of users in the overlapped areas between adjacent BSs increases with the network density. This increase in the number of users aggravates the effect of the coexistence problem, which in turn degrades the network performance.

Figure 10(a) depicts the ratio between the number of served users in the overlapped areas and total number of users in these overlapped areas for the compared schemes (we denote this ratio as $\gamma$ ). This figure shows that both FDFH and SDFH outperform DFH in terms of $\gamma$, as both schemes (FDFH and SDFH) aim at reducing the chances of using same working channels for adjacent WRAN cells. This reduction in turn results in lower number of collisions in the overlapped areas compared with DFH. The impact of this effect clearly appears in the case of FDFH under small numbers of users, as the available channels can satisfy most of the users' requests with small chances of collisions between adjacent cells. Regarding the performance of FSDFH, Figure 10(a) shows that this scheme almost always achieves higher performance than FDFH and SDFH. This is because FSDFH collectively exploits the strengths of both schemes (FDFH and SDFH). The CDFH scheme outperforms all other schemes, specifically at high numbers of system users. This is attributed to the dynamic and cooperative nature of $\mathrm{CDFH}$ in choosing working channels for adjacent BSs, which allows for channel reuse. At low numbers of system users, FDFH, CDFH, and FSDFH approximately achieve the same performance. This comes from the fact that these three schemes succeed in choosing different working channels for adjacent BSs.

We now compare the BER and throughput for the proposed schemes in Figures 10(b) and (c), respectively. In these figures, we only consider collisions between adjacent BSs, i.e., a collision occurs when a channel is simultaneously used to serve several users (which belong to different BSs) in an overlapped area. These figures show that our proposed schemes significantly reduce the BER and improve the throughput relative to DFH under all tested numbers of users. These results are in line with those in Figure 10(a). CDFH results in zero BER (assuming only collisions between overlapped cells) and the highest throughput among all compared schemes. This is attributed to the cooperative nature of CDFH, i.e., it avoids using same working channels for overlapped cells. 


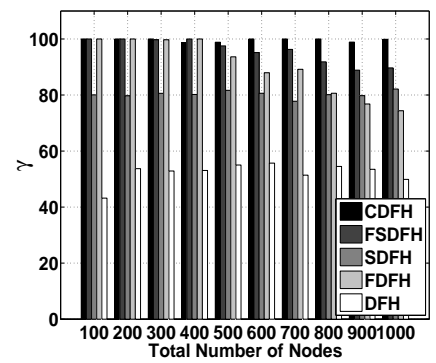

(a) $\gamma$.

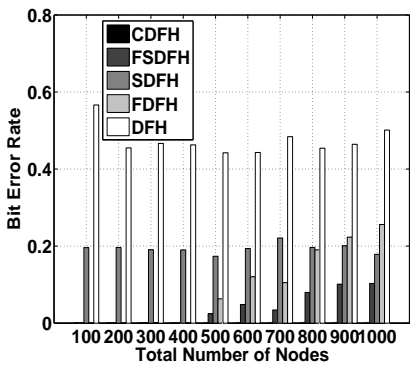

(b) BER.

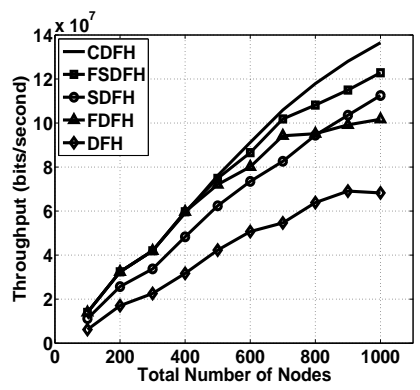

(c) Throughput.

Fig. 10. Performance of the proposed schemes versus total number of nodes (sparse topology).

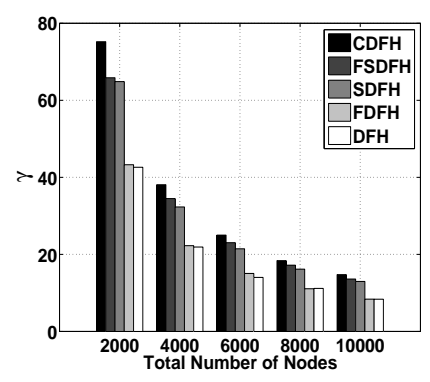

(a) $\gamma$.

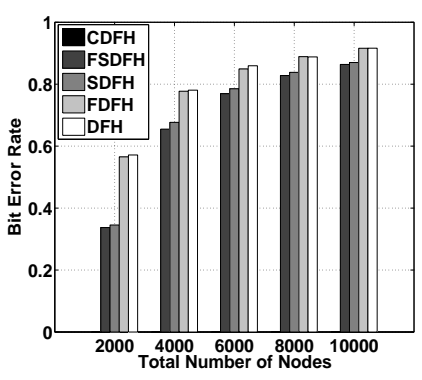

(b) BER.

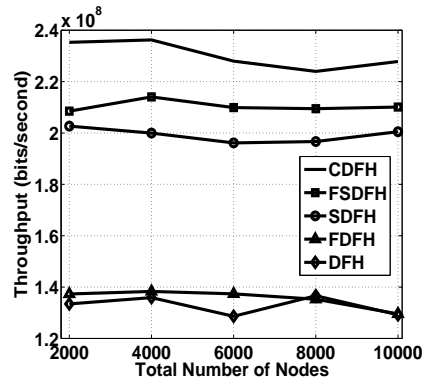

(c) Throughput.

Fig. 11. Performance of the proposed schemes versus total number of nodes (dense topology).

2) Performance under a Dense Network: In this case, we consider a dense topology, where the total number of system users ranges from 2000 to 10000 users. Figures 11(a), (b), and (c) reveal that the compared schemes generally achieve similar performance under sparse and dense topologies. However, in the case of a dense network, the proposed schemes achieve smaller values of $\gamma$ and higher values of BER than these in the case of a sparse network. These are expected results due to the fact that the coexistence problem aggravates with the network density. Note that in Figure 11(c), the shown throughput reflects the performance under the saturation case. This saturation occurs because both the number of available channels and the capacity per channel are limited.

\section{CONClusions And Future Work}

In this paper, we proposed four schemes for IEEE 802.22 WRAN systems, coined FDFH, SDFH, CDFH, and FSDFH. These schemes address one of the main concerns for WRAN systems, namely coexistence problem. The main goal of the proposed schemes is to provide the users in the overlapped areas between adjacent WRAN cells with the requested services without affecting other users' services. These schemes differ from each other in the way of selecting working channels. In particular, FDFH determines a fixed schedule for selecting the next working channel. CDFH cooperatively selects working channels. SDFH divides a cell into sectors to decrease the chances of having several WRAN users in overlapped sectors between neighboring BSs that are served by same operating channels. FSDFH exploits the advantages of FDFH and SDFH with no additional overhead. We studied the performance of the four proposed schemes via simulations, and compared it with that of the fundamental operation mode in WRAN systems (DFH). The results showed that the proposed schemes outperform the DFH in terms of throughput, number of served users, and BER.

As a future work, we plan on designing a comprehensive WRAN system. The basic idea of this design is to incorporate the main features of the proposed schemes into a novel scheme that results in low overhead complexity and high network performance. Other aspects for our future work include distributed/centralized sensing, user detection, dynamic spectrum sharing, etc.

\section{REFERENCES}

[1] http://www.ieee802.org/22/, IEEE 802.22 WRAN website.

[2] W. Hu, D. Willkomm, L. Chu, M. Abusubaih, J. Gross, G. Vlantis, M. Gerla, and A. Wolisz, "Dynamic frequency hopping communities for efficient IEEE 802.22 operation," IEEE Communications Magazine, vol. 25, no. 5, pp. 80-87, May 2007.

[3] Cognitive Wireless RAN Medium Access Control (MAC) and Physical Layer (PHY) Specifications: Policies and Procedures for Operation in the TV Bands, IEEE P802.22/D0.1 Draft Standard for Wireless Regional Area Networks - Part 22.

[4] C. Stevenson, C. Cordeiro, E. Sofer, and G. Chouinard, "Functional requirements for the 802.22 WRAN standard r47," Jan. 2006.

[5] D. Grandblaise and W. Hu, "Inter base stations adaptive on demand channel contention for IEEE 802.22 WRAN self coexistence," Technical proposal submitted to the IEEE $802.22 \mathrm{WG}$, Jan. 2007.

[6] C. Cordeiro, K. Challapali, D. Birru, and S. Shankar, "IEEE 802.22: The first worldwide wireless standard based on cognitive radios," in Proceedings of the First Symposium on New Frontiers in Dynamic Spectrum Access Networks (DySPAN), November 2005.

[7] IEEE 802.22 Working group, WRAN Reference Model, Doc. Num. 22-04-0002-12-0000.

[8] K. Challapali, D. Birru, and S. Mangold, "Spectrum agile radio for broadband applications," EE Times In-Focus, Aug. 2004.

[9] http://datasheets.maxim-ic.com/en/ds/MAX2820-MAX2821A.pdf.

[10] Mesquite Software Incorporation, http://www.mesquite.com. 